\title{
Perfil dos egressos e da produção dos Trabalhos de Conclusão de Curso de uma especialização em saúde mental: análise quanti-qualitativa entre 2011-2019
}

Recebido: 17 mar 2021

Revisado: 19 abr 2021

Aceito: 26 abr 2021

Autor de correspondência: Ivonice Siqueira de Sá ivonicesiqueira@hotmail.com

Como citar: Sá IS, Salgado CL, Garcia PT, Oliveira AEF, Pinho JRO. Perfil dos egressos e da produção dos trabalhos de conclusão de curso de uma especialização em saúde mental: análise quantiqualitativa entre 2011-2019. J Manag Prim Health Care. 2021;13:e04. https://doi.org/ jmphc.v13.1120.

Conflito de interesses: Os autores declaram não haver nenhum interesse profissional ou pessoal que possa gerar conflito de interesses em relação a este manuscrito.

Copyright: Este é um artigo de acesso aberto, distribuído sob os termos da Licença Creative Commons (CC-BY$\mathrm{NC}$ ). Esta licença permite que outros distribuam, remixem, adaptem e criem a partir do seu trabalho, mesmo para fins comerciais, desde que the atribuam o devido crédito pela criação original.

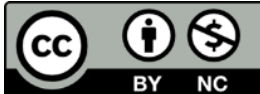

Perfil de los egresados y elaboración de los trabajos de conclusión de una especialización en salud mental: análisis cuanti-cualitativo entre 2011-2019

Profile of graduates and the production of the conclusion papers of a specialization in mental health: quanti-qualitative analysis between 2011-2019

Ivonice Siqueira de $S^{(1)}$ Christiana Leal SALGADO(2) Paola Trindade GARCIA ${ }^{(2)}$ Ana Emília Figueiredo de OLIVEIRA ${ }^{(2)}$ Judith Rafaelle Oliveira PINHO(2)

\footnotetext{
(1) Prefeitura Municipal de São Luis, MA Brasil.

(2) Universidade Federal do Maranhão, São Luis, MA, Brasil.
}

\section{Resumo}

O estudo teve como objetivo analisar o perfil dos egressos e a produção dos trabalhos de conclusão de curso de um curso de especialização em saúde mental na modalidade Educação à Distância ofertado pela UNA-SUS/UFMA. Foi realizada uma pesquisa descritiva de caráter quantitativo e qualitativo cujas fontes de dados foram os trabalhos de conclusão de curso da especialização, as fichas de matrícula e o banco de histórico de vínculo do Cadastro Nacional de Estabelecimentos de Saúde de todos os egressos do curso de especialização em saúde mental da UNA-SUS/UFMA. A coleta de dados aconteceu de agosto de 2018 a maio de 2019, de forma retrospectiva e prospectiva. As análises quantitativa e qualitativa foram realizadas através dos softwares Stata $₫ 14$ e Open EVOC $₫$, respectivamente. A maioria dos egressos eram mulheres $(77,82 \%)$, graduados em enfermagem $(50,58 \%)$, atuavam na área de saúde mental, porém não tinham formação prévia na área. Identificou-se alta rotatividade de locais de trabalho comparando os anos de 2011 e 2019 e que muitos profissionais estão fora do mercado de trabalho. Os descritores dos TCC que compõem o núcleo central da análise qualitativa foram: transtorno mental (frequência: 16,08\% / ordem: 1,65), saúde mental (frequência: 15,43\% / ordem: 1,83) e depressão (frequência: 5,45\% / ordem: 1,19), refletindo os principais contextos vivenciados no trabalho dos discentes. Ofertas de educação permanente como o curso de especialização em saúde mental são estratégias importantes para a promoção e manutenção do cuidado em saúde mental norteado pelo princípio da integralidade, da humanização e do respeito ao sujeito na sua singularidade, ao mesmo tempo, rompe os resquícios da psiquiatria clássica centrada na doença.

Descritores: Educação em Saúde; Saúde Mental; Educação à Distância; Especialização. 


\title{
Resumen
}

El estudio tuvo como objetivo analizar el perfil de los egresados y la producción del trabajo de conclusión de un curso de especialización en salud mental en la modalidad de Educación a Distancia que ofrece UNA-SUS / UFMA. Se realizó una investigación descriptiva cuantitativa y cualitativa, cuyas fuentes de datos fueron los trabajos de conclusión del curso de especialización, las hojas de matrícula y el banco histórico de enlaces del Registro Nacional de Establecimientos de Salud de todos los egresados de un curso de especialización en Salud Mental de la UNA -SUS / UFMA. La recolección de datos se llevó desde agosto de 2018 a mayo de 2019, de manera retrospectiva y prospectiva, el análisis cuantitativo se realizó utilizando el software Stata ${ }^{\circledR} 14$, el análisis cualitativo con el software Open EVOC $\AA$. La mayoría de los egresados eran mujeres (77,82\%), licenciados en enfermería (50,58\%), trabajaban en el área de salud mental, pero no tenían formación previa en el área, hubo una alta rotación de puestos de trabajo comparando los años de trabajo. 2011 y 2019, muchos profesionales están fuera del mercado laboral, los descriptores de la TCC que conforman el núcleo central del análisis fueron: trastorno mental (frecuencia: 16,08\% / orden: 1,65), salud mental (frecuencia: 15,43\% / orden: 1,83) y depresión (frecuencia: 5,45\% / orden: 1,19), reflejando los principales contextos vividos en el trabajo. Las ofertas de educación permanente como el curso de especialización en salud mental son estrategias importantes para la promoción y mantenimiento de la atención en salud mental guiados por el principio de integralidad, humanización y respeto al individuo en su singularidad, rompiendo al mismo tiempo los remanentes de psiquiatría clásica centrada en la enfermedad.

Palabras-claves: Educación en Salud; Salud Mental; Educación a Distancia; Especialización.

\begin{abstract}
The aim was to profile of the graduates and the production of the conclusion work of a specialization course in mental health in the Distance Education modality offered by UNA-SUS / UFMA. Was carried out a quantitative and qualitative descriptive research, data sources were the completion of course work (CCW) in Mental Health offered by UNA-SUS / UFMA, the registration forms and the National Register of Health Establishments of all graduates of a specialization course. Data collection occurred from August 2018 to May 2019, retrospectively and prospectively. The quantitative analysis was performed using the software Stata ${ }^{\circ} 14$, and qualitative analysis with Open EVOC $₫$ software. Most graduates were women $(77.82 \%)$, graduated in nursing $(50.58 \%)$, worked in the mental health services, but had no previous training in the area, was detected a high turnover of workplaces comparing the years 2011 and 2019, many professionals are out of the job market, the CCW descriptors that make up the central core of the analysis were: mental disorder (frequency: $16.08 \%$ / order: 1.65), mental health (frequency: 15, 43\% / order: 1.83) and depression (frequency: 5.45\% / order: 1.19), reflecting the main contexts experienced at work. Health permanent education offers such as the specialization course in mental health are important strategies for the promotion and maintenance of mental health care guided by the principle of integrality, humanization and respect for the individual in his / her uniqueness, at the same time breaking the remnants of psychiatry classic disease-centered.
\end{abstract}

Keywords: Health Education; Mental Health; Education, Distance; Specialization.

\section{Introdução}

A Política Nacional de Educação Permanente em Saúde - PNEPS instituída pela portaria n. 198, e reformulada em 2007, pela Portaria n. 1.996 foi criada com o propósito regulamentar e garantir a oferta de educação permanente em saúde (EPS) aos profissionais do Sistema Único de Saúde - SUS. ${ }^{1}$ Assim, a formação dos trabalhadores do SUS faz parte da agenda governamental e, desse modo, os gestores têm procurado influenciar em um novo perfil profissional, inserindo variadas iniciativas programáticas integrando educação, trabalho, gestão e organização dos serviços, com o intuito de que mudanças na formação modifiquem as práticas de saúde. ${ }^{2}$ 
ISSN 2179-6750

A educação permanente em saúde (EPS) é uma estratégia de aprendizagem no trabalho que acontece no espaço das organizações, incorporando a lógica do ensinoaprendizagem significativo, potencializando a transformação de práticas profissionais. ${ }^{3}$ Uma das principais estratégias para viabilizar a PNEPS, tem sido a Educação a Distância (EaD) pois permite a formação de profissionais de forma rápida e flexível. Essa metodologia de ensino é norteada pela concepção de sala de aula ampliada, onde espaço físico e tempo determinados são substituídos pela mediação tecnológica utilizando-se recursos da tecnologia de informação e comunicação (TIC) para superar a distância espaço-temporal. ${ }^{4}$

Com essa estratégia é possível o alcance de um número expressivo de profissionais que atuam distantes de grandes centros, através da oferta de cursos que podem ser acessados em diversos espaços e em tempos diferentes, tornando possível a flexibilização e o acesso ao conhecimento por meios de recursos didáticos disponíveis, efetivando a democratização do ensino aprendizagem. ${ }^{5}$

Em 2010, o decreto n. 7.385, de 8 de dezembro de 2010, instituiu a Universidade Aberta do SUS - UNA-SUS com a finalidade de atender às necessidades de capacitação e educação permanente dos trabalhadores do SUS, por meio do desenvolvimento da modalidade de educação a distância na área da saúde. ${ }^{6}$

Até março de 2021 a Secretaria executiva do sistema UNA-SUS registrou 4.598.816 matrículas em cursos ofertados, contemplando profissionais que atuam em 5.568 municípios, abrangendo 27 unidades da federação, 435 regiões de saúde e 1.041 ofertas educacionais. ${ }^{7}$ Dentre os vários cursos ofertados, desde o início da rede, foi registrado somente uma única oferta de especialização em saúde mental entre os anos de 2011 e 2012 em parceria com a Universidade Federal do Maranhão - UFMA, sendo considerado o primeiro na área de saúde mental da rede UNA-SUS. ${ }^{7}$

O curso de especialização foi parte integrante de um projeto de qualificação em saúde mental de abrangência nacional que incluía também cursos de capacitação. Para a especialização foram destinadas quinhentas vagas e o público-alvo eram os profissionais da área da saúde de nível superior, vinculados à Rede de Assistência à Saúde Mental do SUS, a Estratégia Saúde da Família - ESF e Núcleo Ampliado de Saúde da Família - NASF. ${ }^{7}$

A realização do curso de especialização em saúde mental (ESM) pela UNA-SUS/UFMA foi pautada na relevância do cuidado em saúde mental para a saúde global e a alta prevalência de transtornos mentais causadores de sofrimento e morbidade, que imprime a necessidade de cuidados qualificados e habilidades dos profissionais. Desse modo, compreende-se que os principais pontos abordados na construção dos trabalhos de conclusão de curso desse projeto pioneiro, seriam importantes para direcionar novas ações formativas na mesma área. 
ISSN 2179-6750

A relevância científica e social se traduz pela possibilidade de conhecer a arena real onde os processos de trabalho são construídos e/ou desconstruídos e esse conhecimento permitir agregar possibilidades elucidativas e interventivas bem como potencializar a garantia de atenção integral, resolutiva e humanizada as famílias através da formação profissional voltada para a saúde mental. Há necessidade de investimento em novos estudos que possam responder às inquietações e aos desafios do trabalho para melhor conhecer, acompanhar e avaliar as iniciativas que ocorrem na educação no mundo do trabalho da saúde, particularmente no âmbito do SUS. ${ }^{2}$

Desse modo, essa pesquisa teve como objetivo analisar o perfil dos egressos e a produção dos trabalhos de conclusão de curso de uma especialização em saúde mental na modalidade de Educação à Distância ofertada pela UNA-SUS/UFMA.

\section{Metodologia}

\section{Desenho do estudo}

Foi realizado uma pesquisa descritiva de caráter quantitativo e qualitativo cujas fontes de dados foram os trabalhos de conclusão de curso da especialização em Saúde Mental ofertada pela UNA-SUS/UFMA, as fichas de matrícula e o banco de histórico de vínculo do Cadastro Nacional de Estabelecimentos de Saúde de todos os egressos do curso de especialização em saúde Mental da UNA-SUS/UFMA.

A opção por agregar dois métodos para análise dos dados se deu pela possibilidade de ampliar o olhar para além das características dos egressos, mas também para o que foi produzido pelos discentes como produto de suas aproximações com sua realidade de trabalho e problemas enfrentados no cotidiano.

\section{Descrição do curso}

O curso teve carga horária de 420 horas, divididas em quatro eixos temáticos:

- $\quad$ Eixo I - Introdução ao Curso de Saúde Mental

- $\quad$ Eixo II - Clínica e Atenção Psicossocial

- $\quad$ Eixo III - Intervenção

- $\quad$ Eixo IV - Trabalho de Conclusão de Curso

Cada eixo foi constituído por módulos de aprendizagem (Quadro 1). Cada módulo ofereceu conteúdos e atividades que asseguraram a coerência teórico-metodológica necessária à qualificação profissional do discente, tendo por objetivo desenvolver as competências necessárias à sua formação como Especialista em Saúde Mental. Vale ressaltar que a inscrição do aluno nesse curso estava atrelada a apresentação de anuência do gestor para fins de comprovação de vínculo com o SUS. 


\begin{tabular}{|c|c|}
\hline +/+Núcleo central & +/-Primeira periferia \\
\hline OME*: < média (maior importância) & OME: = > média (menor importância) \\
\hline Frequência: alta & Frequência: alta \\
\hline$-/+$ Zona de contraste & -/- Segunda periferia \\
\hline OME< média: maior importância & OME: = > média (menor importância) \\
\hline Frequência: baixa & Frequência: baixa \\
\hline
\end{tabular}

Quadro 1. Representação esquemática do Quadro de Quatro Casas de Vergès Fonte: Elaborado pelos autores (2020).

*OME: ordem média de evocação

As atividades realizadas ao longo do curso objetivaram conduzir o discente a uma (nova) visão da realidade no seu entorno de atuação, ensejando o desenvolvimento de uma prática assistencial com qualidade. As turmas foram organizadas conforme o número de discentes, de maneira que, para cada 25 a 30 alunos, tinha um tutor para apoio pedagógico.

Foram programados encontros presenciais obrigatórios, organizados nos polos regionais, para a discussão e o aprofundamento dos conteúdos curriculares, focalizando a contextualização teórica do processo didático-pedagógico, e para a realização de atividades avaliativas presenciais obrigatórias. Os encontros presenciais ocorreram preferencialmente aos sábados.

O acompanhamento no desempenho nas atividades propostas foi realizado pelo tutor a partir de critérios que envolviam a qualidade das atividades, controle de acessos ao Ambiente Virtual de Aprendizagem e pela presença nos encontros.

Para possibilitar uma construção gradual e progressiva do Trabalho de Conclusão de Curso - TCC, o eixo IV da especialização foi desenvolvido transversalmente, ou seja, ao longo do curso e em paralelo com outros módulos (dos eixos I, II e II) - abordou a sistematização da metodologia de pesquisa, dando suporte às atividades que culminaram com a elaboração do TCC.

O TCC foi uma das formas de avaliação, onde cada aluno, individualmente, pôde escolher o formato de elaboração: monografia, trabalho científico para periódico ou plano de ação (projeto de intervenção) para seu município. As diretrizes gerais foram para que o processo de construção dos TCC promovesse uma reflexão sobre a realidade da área de abrangência do discente, que apresentasse indicadores de acompanhamento e avaliação das ações que seriam desenvolvidas, bem como apresentasse alternativas para a melhoria da assistência de sua comunidade. 
Esse desenho pedagógico, associado à estrutura do curso, possibilitou um aprofundamento teórico, valorização do conhecimento já constituído pelos profissionais e estimulação da reflexão sobre a prática assistencial.

\section{Coleta e análise da dados}

Foram incluídos no estudo todos os alunos que concluíram o curso de especialização, cujo TCC tivesse pelo menos 2 descritores no resumo. Não foram incluídos os alunos cujos dossiês de matrícula não foram localizados no acervo da UNA-SUS-UFMA. Resultando no desenho amostral ilustrado na Figura 1.

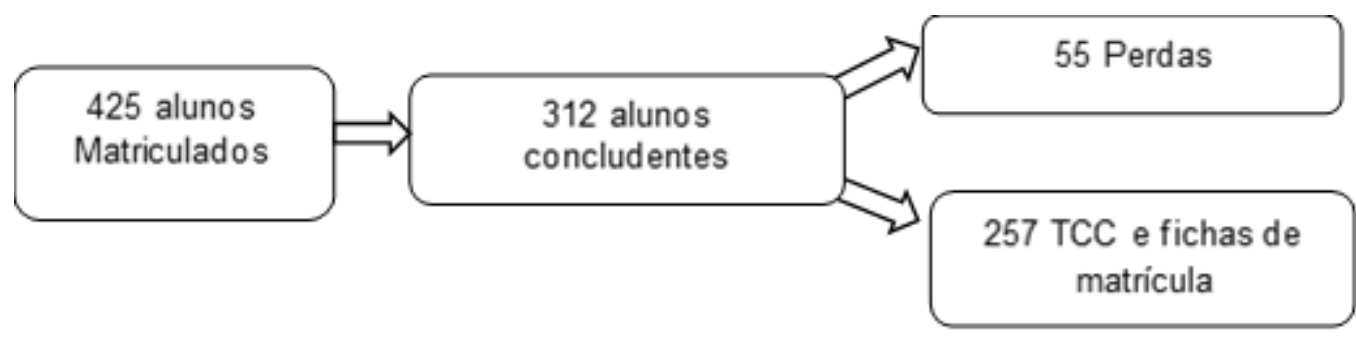

Figura 1. Desenho amostral

Fonte: Elaborado pelos autores (2020).

A coleta de dados aconteceu de agosto de 2018 a maio de 2019 e foi realizada em 3 etapas. As duas primeiras, de ordem quantitativa e a terceira, de caráter qualitativo.

A primeira etapa foi relativa aos dados de caracterização dos participantes onde as seguintes variáveis foram obtidas a partir da ficha de matrícula: local de trabalho, tempo de graduação, tipo de graduação, idade, sexo, estado civil, experiência de formação e de trabalho em saúde mental. Para definição da variável experiência de formação foi considerada a realização de cursos na área de saúde mental, na modalidade de capacitação ou especialização, e para definição de experiência de trabalho a inserção profissional direta em instituições de assistência direta em saúde mental autodeclarada na ficha de inscrição.

A segunda etapa de coleta consistiu na busca de dados na base de histórico de vínculos profissionais no Cadastro Nacional de Estabelecimentos de Saúde - CNES para averiguar em qual município o profissional/aluno se vinculava no momento do curso (qual município o gestor emitiu carta de anuência ao profissional) e qual município esse profissional se encontrava vinculado no momento da coleta.

Para análise dos dados quantitativos da caracterização dos egressos foram verificados frequência, média e desvio padrão no software Stata ${ }^{\circledR}$.

A terceira etapa, a fase qualitativa do estudo, consistiu na análise prototípica dos descritores presentes nos TCC a partir da teoria das representações sociais, ${ }^{8}$ para isso foi 
realizada a lematização, onde os termos são homogeneizados, e a análise prototípica que se baseia no cálculo de frequência e ordem de evocações das palavras e na formulação de categorias e avaliação de suas frequências, composições e co-ocorrências. A frequência e ordem das palavras forneceu duas coordenadas classificadas em valores altos e baixos e quatro zonas que caracterizam a tabela de resultados da análise prototípica. ${ }^{8}$

Para lematização os descritores foram revisados e os sinônimos foram reescritos conforme consta nos descritores em ciências da saúde (DeCS), sem acentos ou pontuações, e agrupados por palavras que compartilhem o mesmo radical e classe para evitar ambiguidades e divergências a respeito da categorização dos conteúdos para homogeneizar as palavras. $^{9}$

A orientação para escolha dos descritores de um trabalho de conclusão de curso na UNA-SUS/UFMA é de palavras indexadas no DeCS que é um vocabulário dinâmico totalizando 33.558 descritores e qualificadores. Existem 7.741 códigos hierárquicos em categorias. As seguintes são categorias DeCS e seus totais de descritores: Ciência e Saúde (231), Homeopatia (1.948), Saúde Pública (3.527) e Vigilância Sanitária (817). ${ }^{10}$ Nesse estudo 711 descritores foram analisados.

A distribuição dos termos nos quadrantes formou as quatro casas: A casa da esquerda superior (núcleo central) onde encontram-se os termos mais frequentes e mais importantes. No quadrante inferior esquerdo (zona de contraste) consta termos de importância, porém menos frequentes no escopo. No quadrante direito superior (primeira periferia) fazem parte os descritores com alta frequência, acima da média e hierarquia de menor importância. No quadrante direito inferior (segunda periferia) consta descritores com baixa frequência, abaixo da média. O Quadro 1 apresenta a representação esquemática do Quadro de Quatro Casas de Vergès. ${ }^{8}$

O nível de importância baseia-se no princípio segundo o qual o quanto antes uma pessoa se lembra de uma palavra, maior é a representatividade dessa palavra num grupo formado por pessoas com perfil semelhante.

\section{Considerações éticas}

A pesquisa faz parte do projeto Cooperação Técnica para a Produção de Objetos de Aprendizagem e Inovação em EaD para os Profissionais do SUS: estudo exploratório sobre ensino e aprendizagem em cursos ofertados aos profissionais de saúde na modalidade EaD e foi aprovado pelo Comitê de Ética em Pesquisa do Hospital Universitário da Universidade Federal do Maranhão/HU/UFMA sob parecer n. 1.376.628. 
ISSN 2179-6750

\section{Resultados}

Observou-se que a maioria dos alunos do curso de especialização em Saúde Mental a distância da UNA-SUS/UFMA foram mulheres (77,82\%), solteiros (61,87\%), com média de idade de 38,5 anos (DV=10.64), graduados em enfermagem (50,58\%), com graduação concluída em 2009 (13,62\%) e não possuíam título de outra especialização (43,19\%), como mostra a Tabela 1.

Tabela 1. Caracterização dos egressos do curso de especialização em Saúde Mental na Modalidade EaD da UNA-SUS/UFMA. Brasil. São Luís - MA. 2019

\begin{tabular}{|c|c|c|c|}
\hline \multicolumn{2}{|c|}{ Variáveis } & \multirow{2}{*}{$\begin{array}{c}\mathrm{n} \\
57\end{array}$} & \multirow{2}{*}{$\begin{array}{c}\% \\
22,18\end{array}$} \\
\hline Gênero & Masculino & & \\
\hline & Feminino & 200 & 77,82 \\
\hline \multirow[t]{3}{*}{ Estado civil } & Solteiro & 159 & 61,87 \\
\hline & Casado & 84 & 32,68 \\
\hline & Outros & 14 & 5,45 \\
\hline \multirow[t]{5}{*}{ Graduação } & Enfermagem & 130 & 50,58 \\
\hline & Psicologia & 31 & 12,06 \\
\hline & Serviço social & 25 & 9,73 \\
\hline & TO* & 17 & 6,61 \\
\hline & Outros & 54 & 21,02 \\
\hline \multirow{5}{*}{$\begin{array}{l}\text { Ano de conclusão } \\
\text { da graduação }\end{array}$} & 2009 & 35 & 13,62 \\
\hline & 2008 & 33 & 12,84 \\
\hline & 2007 & 31 & 12,06 \\
\hline & 2006 & 26 & 10,12 \\
\hline & Outros & 132 & 51,36 \\
\hline \multirow{4}{*}{$\begin{array}{l}\text { Especialização } \\
\text { prévia ao curso }\end{array}$} & ESF & 72 & 28,02 \\
\hline & Psicopedagogia & 12 & 4,67 \\
\hline & Outras & 62 & 24,12 \\
\hline & Não Tem & 111 & 43,19 \\
\hline \multirow{2}{*}{$\begin{array}{l}\text { Formação em } \\
\text { saúde mental }\end{array}$} & Sim & 56 & 21,79 \\
\hline & Não & 201 & 78,21 \\
\hline
\end{tabular}

Fonte: Elaborado pelos autores (2020).

*Terapia Ocupacional

A busca de dados do CNES mostrou que, no ano de 2019, a maioria dos egressos não estava vinculada à algum serviço de saúde (35,02\%), condição que era indispensável para matrícula na especialização. O vínculo de trabalho predominante são os Hospitais (21,40\%), a ESF (14,40\%), os Centros de Atenção Psicossocial - CAPS (11,67\%), Docência (3,23\%), o NASF (4,67\%) e outros (6,61\%), conforme sumarizado na Tabela 2. 
ISSN 2179-6750

Tabela 2. Histórico de Vínculo de egressos do curso de especialização em Saúde Mental na Modalidade EaD da UNA-SUS/UFMA. Brasil. São Luís - MA. 2019

\begin{tabular}{c|c|c|c}
\hline \hline \multicolumn{1}{c|}{ Ano } & Local de atuação & $\mathrm{n}$ & $\%$ \\
\hline \multirow{4}{*}{2011} & ESF $^{*}$ & 114 & 44,36 \\
\cline { 2 - 4 } & CAPS $^{* *}$ & 60 & 23,35 \\
\cline { 2 - 4 } & RASM $^{* \star *}$ & 31 & 12,06 \\
\cline { 2 - 4 } & NASF $^{* * *}$ & 20 & 7,78 \\
\cline { 2 - 4 } & Outros & 32 & 12,45 \\
\hline \multirow{4}{*}{2019} & Sem vínculo & 90 & 35,02 \\
\cline { 2 - 4 } & Hospitais & 55 & 21,4 \\
\cline { 2 - 4 } & ESF $^{*}$ & 37 & 14,4 \\
\cline { 2 - 4 } & CAPS $^{* *}$ & 30 & 11,67 \\
\cline { 2 - 4 } & NASF $^{* * *}$ & 12 & 4,67 \\
\cline { 2 - 4 } & Outros & 33 & 12,84 \\
\hline \hline
\end{tabular}

Fonte: Elaborado pelos autores (2020).

Notas: "Estratégia de Saúde da Família; ${ }^{* *}$ Centros de Apoio Psicossocial; ${ }^{* * *}$ Pontos de apoio à Rede de Atenção em saúde Mental; ${ }^{* * *}$ Núcleo Ampliado de Saúde da Família.

Os resultados relacionados à análise prototípica (qualitativa) dos TCC mostram que os descritores que compõe o núcleo central são: transtorno mental (frequência:16,08\% / ordem: 1,65), saúde mental (frequência: 15,43\% / ordem: 1,83) e depressão (frequência: 5,45\% / ordem: 1,19), conforme demonstra o Quadro 2.

\begin{tabular}{|c|c|c|c|c|c|}
\hline \multicolumn{3}{|c|}{ Núcleo Central ( $1^{\circ}$ quadrante) } & \multicolumn{3}{|c|}{$1^{\text {a }}$ Periferia } \\
\hline \multicolumn{3}{|c|}{++ Frequência $\geq 0.39$ / Ordem $<2$} & \multicolumn{3}{|c|}{ +- Frequência $\geq 3.39$ / Ordem $\geq 2$} \\
\hline $\begin{array}{l}\text { Transtorno } \\
\text { Mental }\end{array}$ & $16,08 \%$ & 1,65 & $\begin{array}{l}\text { Rede de } \\
\text { Assistência à } \\
\text { Saúde Mental }\end{array}$ & $15,82 \%$ & 2,44 \\
\hline Saúde Mental & $15,43 \%$ & 1,83 & $\begin{array}{l}\text { Atenção } \\
\text { Primária em } \\
\text { Saúde }\end{array}$ & $2,72 \%$ & 2,24 \\
\hline \multirow[t]{3}{*}{ Depressão } & \multirow[t]{3}{*}{$5,45 \%$} & \multirow[t]{3}{*}{1,19} & $\begin{array}{l}\text { Qualidade de } \\
\text { vida }\end{array}$ & $2,59 \%$ & 2,9 \\
\hline & & & Adolescência & $1,95 \%$ & 2,6 \\
\hline & & & Fatores de risco & $1,82 \%$ & 2,43 \\
\hline
\end{tabular}




\begin{tabular}{|c|c|c|c|c|c|}
\hline & & & $\begin{array}{l}\text { Terapêutica } \\
\text { medicamentosa }\end{array}$ & $2 \%$ & 2,57 \\
\hline \multicolumn{3}{|c|}{$3^{\mathrm{a}}$ Zona de Contraste } & \multicolumn{3}{|c|}{$2^{a}$ Periferia } \\
\hline \multicolumn{3}{|c|}{ - + Frequência <0.39/ Ordem $<2$} & \multicolumn{3}{|c|}{ - -Frequência <4,43/ Ordem $\geq 2$} \\
\hline $\begin{array}{l}\text { Acidente de } \\
\text { Trânsito }\end{array}$ & $0,26 \%$ & 1,5 & Saúde do trabalhador & $0,26 \%$ & 2,5 \\
\hline $\begin{array}{l}\text { Promoção da } \\
\text { Saúde }\end{array}$ & $0,26 \%$ & 2 & Cônjuges & $0,26 \%$ & 2,5 \\
\hline Fonoaudiologia & $0,13 \%$ & 1 & Gestante & $0,26 \%$ & 2,5 \\
\hline
\end{tabular}

Quadro 2: Análise prototípica dos TCC

Fonte: Elaborado pelos autores (2020).

\section{Discussão}

É impactante observar que os resultados dessa pesquisa mostram que $35,02 \%$ dos egressos dessa formação, não possuíam vínculo de trabalho no SUS em 2019. Em acréscimo, houve uma redução entre os que mantinham vínculos na ESF, nos CAPS e nos NASF, e aumento na atuação em hospitais, que não foi referida em 2011, ano de início da especialização.

O trabalho em Hospitais Universitários foi o mais verificado no CNES, isso pode estar relacionado à reestruturação do modelo de gestão desses hospitais que gerou oferta de grande número de vagas ${ }^{11,12}$ com salários mais atraentes do que o oferecido aos profissionais que atuavam na ESF, no NASF e nos CAPS dos municípios.

Estudo realizado em Belo Horizonte evidenciou que os gestores relatam dificuldade para contratar e manter nos serviços de saúde mental profissionais afinados com o paradigma político-assistencial do município. Para os profissionais essa dificuldade se deve a precarização do trabalho dos técnicos de nível superior, com contratos e salários inadequados, problema destacado por todos os participantes ${ }^{13}$ e essa pode ser uma realidade semelhante entre os egressos do curso.

Essas mudanças de vínculo tem motivação em questões que envolvem a precariedade na contratação, dificuldades de relacionamento político entre profissional e gestor, até questões como a realização profissional nas atividades desenvolvidas no âmbito dessa política. ${ }^{14} \mathrm{O}$ que resulta na perda de profissionais estratégicos, uma vez que capacitados contribuiriam para melhoria das ações em saúde, aumentariam a resolutividade dos serviços e dos programas de saúde mental dos municípios. Essa rotatividade de profissionais, justifica ofertas frequentes de cursos sob a lógica da educação permanente em saúde - EPS. 
A enfermagem foi a categoria profissional predominante no curso, vale lembrar que, a força de trabalho na saúde do profissional de enfermagem se sobrepõe às demais profissões, tanto em oferta de trabalho como em ofertas de vagas nas universidades. ${ }^{15}$ Estudo realizado para analisar o design da sala de aula virtual para o novo perfil do aluno EaD foi semelhante a este com predominância de mulheres e enfermeiros. Tendo a Enfermagem maior quantidade de participantes do sexo feminino, $84 \%{ }^{16}$ o que corrobora os resultados dessa pesquisa.

No curso de especialização em saúde mental 78,21\% dos profissionais não possuíam outros cursos de formação em saúde mental embora atuassem nos serviços de saúde entre eles os CAPS, o que não sugeria uma incapacidade de atuação nesse campo, mas que a formação institucionalizada em saúde mental era incipiente.

A Política Nacional de Saúde Mental Brasileira instituída em 2001 já previa mudanças teórico-conceituais, técnico-assistencial, jurídico-política e sociocultural, porém, após vinte anos da reforma psiquiátrica, ainda existe um descompasso na formação em saúde mental ${ }^{17}$ em âmbito nacional e internacional que o país vem se esforçando para superar. ${ }^{18}$

No entanto, vale destacar que o cenário atual é de mudanças na Política de Saúde Mental. O contexto que incorpora à rede as Comunidades Terapêuticas e as Equipes Multiprofissionais Especializadas em Saúde Mental e o Hospital Dia, também apresenta redução do financiamento para os CAPS e aumento em $60 \%$ os recursos aos hospitais Psiquiátricos. ${ }^{19}$ Segundo os idealizadores a proposta da nova política é alcançar uma demanda reprimida de pessoas com transtornos mentais que não se encaixam na Atenção Básica e no CAPS e que desenvolverão suas atividades assistenciais integrada a estes, e o retorno à hospitalização para usuários com transtornos mentais em hospitais psiquiátricos, e, em Comunidades Terapêuticas. ${ }^{20}$

Nos cursos de graduação das áreas da saúde impera a especialização precoce dos alunos constituindo uma defasagem entre o que se ensina na universidade e as especificidades, demandas e necessidades dos sujeitos nos territórios. ${ }^{21}$

A oferta de cursos alinhados à educação permanente em saúde, cuja formação enseja o enfrentamento dos problemas de saúde da população e o desenvolvimento do próprio SUS, ${ }^{22}$ pôde ser verificada na análise dos descritores presentes nos TCC produzidos pelos alunos. Os termos "transtorno mental", "saúde mental" e "depressão" que representam o núcleo central, foram as temáticas mais frequentes e coincidem com os indicadores de saúde mental mais prevalentes no país, que por sua vez, podem estar concebendo a realidade vivenciada no processo de trabalho dos discentes do curso.

Em 2008 a Organização Mundial de Saúde - OMS já previa que, em 2020, a depressão seria a segunda mais importante causa de incapacidade. ${ }^{23}$ Os transtornos mentais 
ISSN 2179-6750

representam uma parcela significativa da demanda da APS, o que pode corroborar os resultados que constituem o núcleo central, já que o transtorno mental comum pode representar de $30 \%$ a $50 \%$ dessa demanda. ${ }^{24}$

Estudos no Brasil e no mundo revelam a limitação das práticas de cuidado em saúde mental ocasionadas pela carência de conhecimento e falta de aproximação dos profissionais com a saúde mental. As equipes não sabem como lidar com as demandas de saúde mental ou não reconhecem suas práticas cotidianas como parte do cuidado em saúde mental em razão da formação profissional, ${ }^{25}$ o que pode estar relacionado aos resultados desta pesquisa, uma vez que os egressos foram estimulados a construir seus trabalhos a partir de problemáticas vivenciadas no cotidiano do trabalho. Nesse contexto, a educação permanente revela-se como importante estratégia para reverter esse panorama, uma vez que pode corrigir problemas como a ausência de atualização e promover modificações na assistência à saúde mental. ${ }^{26}$

Na zona de contraste encontram-se os termos "acidente de trânsito", "promoção da saúde", "fonoaudiologia", que representam assuntos de grande importância, porém para um quantitativo menor de sujeitos. Os termos desse quadrante são complementares ao núcleo central, podendo auxiliar sua na compreensão. ${ }^{8}$

Os quadrantes do lado direito, que compõem a periferia da representação e auxiliam a compreensão do sistema central, também estão mais ligados à prática dos sujeitos. No quadrante superior direito encontra-se o termo "Rede de Assistência em Saúde Mental", "Atenção Primária a Saúde", "qualidade de vida", "adolescência" e "fatores de risco". Estes são considerados termos importantes por sua elevada frequência e menor importância atribuída pelos sujeitos dentro da totalidade de termos.

A Reforma Sanitária e Psiquiátrica trouxe a necessidade do trabalho articulado, há particularidades e complexidades das pessoas com transtornos mentais que não podem ser solucionadas pelos moldes tradicionais de assistência em saúde mental. A proposta requer atenção em equipe multiprofissional, integralidade, responsabilização da equipe vinculada a uma comunidade, intersetorialidade e integração da rede do nível primário ao especializado $^{24}$ e a forma que os descritores aparecem na análise prototípica parecem confirmar essa necessidade.

No entanto, o novo cenário político oferece risco a reforma psiquiátrica, uma construção sólida, que desenvolveu estratégias substitutivas a internação psiquiátrica nos últimos dezoito anos. ${ }^{18}$ Embora no país já exista um vasto movimento de luta, cuja trincheira de resistência está nos serviços territoriais que se insere a Atenção Básica ${ }^{23}$, essas mudanças comprometem a formação dos profissionais, uma vez há retrocesso no modelo assistencial. 
ISSN 2179-6750

Essa disposição dos descritores também pode ser reflexo de que os profissionais das equipes da ESF se sentem despreparados para lidar com a saúde mental, desenvolvendo um cuidado ainda muito centrado na medicação e em encaminhamentos para serviços especializados. Para muitos profissionais a proposta do apoio matricial não está clara, por não terem participado de sua construção. Com isso a ampliação da oferta de cuidados em saúde mental na APS não é bem aceita, sendo caracterizada por estes como 'acréscimo' de trabalho e uma 'não responsabilização' da Atenção Especializada. ${ }^{24}$

Estudo realizado no Nordeste evidenciou que além do apoio matricial, é necessária a existência das Redes de Serviços em saúde mental (CAPS, ambulatórios especializados e residências terapêuticas e outras), bem como a articulação com dispositivos da justiça, cultura, visando ações intersetoriais ${ }^{24}$ que é um descritor que aparece com grande frequência na análise prototípica.

A importância dada a Rede de Atenção em Saúde e a Atenção Primária a Saúde apresentadas nos TCC, também pode refletir o destaque à importância da interlocução entre esses setores possibilitando a criação de espaços de discussão, troca de saberes e reflexão para a prática. Essa necessidade será ainda mais visível diante da manutenção de hospitais psiquiátricos e comunidades terapêuticas, cujo modelo de assistência é baseado em propostas higienistas que fere os direitos dos usuários, excluindo-os do convívio familiar. ${ }^{25}$

A segunda periferia, traz os termos "saúde do trabalhador", "cônjuges" e "gestante" que são de baixa frequência e importância. Destaca-se o termo "saúde do trabalhador" uma vez que os transtornos mentais podem estar relacionados ao trabalho dos profissionais de saúde e a manutenção da saúde mental é necessária tanto para se manter no mercado de trabalho quanto para o exercício da profissão com qualidade.

As transformações na formação em saúde, no trabalho e na gestão são indissociáveis das transformações nos processos de subjetivação e de cuidados em saúde. A formação tem a potencialidade de possibilitar novas práticas organizacionais e de cuidado em saúde, promovendo transformações de atitudes, crenças, conhecimentos e habilidades colaborativas. $^{26}$

A impossibilidade de apresentar informações acerca da transformação da assistência ofertada por estes profissionais após o término do curso, é um fator limitante, pois a qualidade do cuidado não resulta exclusivamente da formação profissional, mas da produção de subjetividade. ${ }^{25}$ Portanto, o desenvolvimento de novas pesquisas para averiguar as potencialidades do cuidado em saúde mental destes profissionais é fundamental para responder algumas perguntas que surgiram no desenvolvimento desse trabalho. 
ISSN 2179-6750

Aponta-se como potencialidade dessa pesquisa a possibilidade de identificação de novas necessidades de formação em saúde mental com vistas a potencial interlocução da saúde mental com a atenção básica, da necessidade do cuidado em rede considerando a integralidade do indivíduo. Em adição, esta pesquisa permitiu conhecer a atual inserção profissional desses egressos no SUS.

\section{Conclusão}

Ofertas de educação permanente como o curso de especialização em saúde mental são estratégias importantes para a promoção e manutenção do cuidado em saúde mental norteado pelo princípio da integralidade, da humanização e do respeito ao sujeito na sua singularidade, ao mesmo tempo, rompe os resquícios da psiquiatria clássica centrada na doença. No entanto, a rotatividade de profissionais interfere negativamente nesse processo, impede a formação de vínculos e compromete a longitudinalidade do cuidado.

A desvalorização profissional apontada na literatura como fator gerador da rotatividade pode deixar profissionais capacitados fora do mercado de trabalho, ao passo que outros estão assumindo os serviços e não se sabe se esses estão preparados para a assistência em saúde mental. A pesquisa foi direcionada a uma realidade não esperada e traz resultados significativos para formulação de novos processos formativos.

Os termos apresentados nos TCC reforçam a necessidade de cuidados em saúde mental em rede e a importância da APS e, são coerentes com as questões centrais da literatura científica da área, que demostra que as pessoas com transtornos mentais buscam assistência na APS mais não encontram o amparo necessário, resultando em um hiato terapêutico. Outros estudos poderão complementar os achados referentes ao impacto da assistência em saúde mental nos municípios em que atuam os profissionais que realizaram o curso.

Espera-se que os resultados obtidos com a pesquisa ofereçam subsídios que justifiquem a necessidade de novas formações em saúde mental, que sejam contínuas e permanentes, pela necessidade urgente da assistência em saúde mental de base comunitária, territorial, integral e humanizada.

\section{Contribuição autoral}

I. S. de Sá realizou a escrita, a análise, interpretação dos dados dos dados. C. L. Salgado realizou interpretação dos dados e revisou o texto completo. P. T. Garcia realizou escrita, avaliação dos dados, revisão do texto. A. E. M de Oliveira realizou análise, interpretação dos dados dos dados, revisou o texto completo e aprovação do formato 
final. J. R. O. Pinho realizou a escrita, análise, interpretação dos dados dos dados, revisou o texto completo e aprovação do formato final.

\section{Referências}

1. Ministério da Saúde (BR). Política nacional de educação permanente em saúde. Brasília, DF; 2009. (Série B. Textos básicos de saúde; Série pactos pela saúde; vol. 9).

2. Leite $C M$, Pinto ICM, Fagundes TLQ. Educação permanente em saúde: reprodução ou contra-hegemonia? Trab Educ Saude. 2020;18(suppl 1):1-15. https://doi.org/10.1590/1981-7746-sol00250.

3. Oliveira AEF, Ferreira EB, Sousa RR, Castro Júnior EF, Maia MFL. Educação a distância e formação continuada: em busca de progressos para a saúde. Rev Bras Educ Med. 2013;37(4):578-83. https://doi.org/10.1590/S0100-55022013000400014.

4. Cezar DM, Costa MR, Magalhães CR. Educação a distância como estratégia para a educação permanente em saúde?. EmRede. 2017;4(1).

5. Ministério da Educação (BR). Portaria Interministerial n. 10, 11 julho 2013. Regulamenta o Decreto n. 7.385, 8 dezembro 2010, que instituiu o Sistema Universidade Aberta do Sistema Único Saúde (UNA-SUS) [Internet]. [Brasília, DF: Ministério da Educação]; 2013 [citado 9 ago. 2020]. Disponível em: https://ares.unasus.gov.br/acervo/handle/ARES/3756

6. Ministério da Saúde (BR). UNA-SUS em números [Internet]. [Brasília, DF]: MS; 2021 [citado 9 mar. 2021]. Disponível em: https://www.unasus.gov.br/numeros/arouca

7. Wachelke J, Wolter R. Critérios de construção e relato da análise prototípica para representações sociais. Psicol Teor Pesqui. 2011;27(4):521-6. https://doi.org/10.1590/S0102-37722011000400017.

8. Descritores em Ciencias da Saúde: Decs: 2017 [Internet]. São Paulo: BIREME. 2017 [citado 1 out. 2019]. Disponível em: http://decs.bvsalus.org/

9. Barros RT. Modelos de gestão na administração pública brasileira: reformas vivenciadas pelos hospitais universitários federais. Rev Mestrados Profissionais. 2013;2(1):252-80. https://doi.org/10.52614/2317-0115.2013.325.

10. Sodré F, Littike D, Drago LMB, Perim MCM. Empresa Brasileira de serviços hospitalares: um novo modelo de gestão?. Serv Soc Soc. 2013;(114):365-80. https://doi.org/10.1590/S0101-66282013000200009. 
11. Lima ICBF, Passos ICF. Residências Integradas em saúde mental: para além do tecnicismo. Trab Educ Saude. 2019;17(2):e0020940. https://doi.org/10.1590/1981-7746-sol00209.

12. Medeiros CRG, Junqueira GAW, Schwingel G, Carreno I, Jungles LAP, Saldanha OMFL. A rotatividade de enfermeiros e médicos: um impasse na implementação da estratégia de saúde da família. Cienc Saude Colet. 2010;15(suppl 1):1521-31. https://doi.org/10.1590/S1413-81232010000700064.

13. Instituto Nacional de Estudos e Pesquisas Educacionais Anísio Teixeira. Sinopses estatísticas da educação superior: graduação [Internet]. Brasília, DF: INEP; 2019 [citado 23 set. 2019]. Disponível em: http://inep.gov.br/sinopses-estatisticas-da-educacao-superior

14. Souza Junior TG, Rodrigues GOCA. Design da sala de aula virtual para o novo perfil do aluno de EaD. Ead Foco. 2018;8(1):1-12. https://doi.org/10.18264/eadf.v8i1.698.

15. Onocko-Campos R, Emerich BF, Ricci EC. Residência multiprofissional em saúde mental: suporte teórico para o percurso formativo. Interface (Botucatu). 2019;23:e17081.3. https://doi.org/10.1590/Interface.170813.

16. Delgado PG. Reforma psiquiátrica: estratégias para resistir ao desmonte [Editorial]. Trab Educ Saude. 2019;17(2):4-7. https://doi.org/10.1590/1981-7746-sol00212.

17. Ministério da Saúde (BR). Nota técnica n. 11/2019. Esclarecimentos sobre as mudanças na política nacional de saúde mental e nas diretrizes na política nacional sobre drogas. Brasília, DF: MS; 2019.

18. Pitta AMF, Guljor AP. A violência da contrarreforma psiquiátrica no Brasil: um ataque à democracia em tempos de luta pelos direitos humanos e justiça social. Cad CEAS. 2019;(246):6-14. http://dx.doi.org/10.25247/2447-861X.2019.n246.p6-14.

19. Emerich F, Onocko-Campos R. Formação para o trabalho em saúde mental: reflexões a partir das concepções de sujeito, coletivo e instituição. Interface (Botucatu). 2019;23:e1705. https://doi.org/10.1590/Interface.170521.

20. Ivbjaro G, Kolkiewicz L, Lionis C, Svab I, Cohen A, Sartorius N. Primary care mental health and Alma-Ata: from evidence to action. Ment Health Fam Med. 2008;5:67-70.

21. Gryschek G, Pinto AAM. Saúde mental: Como as equipes de saúde da família podem integrar esse cuidado na atenção básica?. Cienc Saude Colet. 2015;20(10):3255-62. https://doi.org/10.1590/1413-812320152010.13572014. 
22. Pini JS, Waidman MAP. Fatores interferentes nas ações da equipe da estratégia saúde da família ao portador de transtorno mental. Rev Esc Enferm USP. 2012;46(2):372-9. https://doi.org/10.1590/S0080-62342012000200015.

23. Santos KL, Surjus LTLS. Ativando as engrenagens da educação permanente na rede de atenção psicossocial: os desafios no cuidado a pessoas em abuso de substâncias psicoativas. Interface (Botucatu). 2019;23:e180299. https://doi.org/10.1590/Interface.180299.

24. Sousa FSP, Jorge MSB, Vasconcelos FMG, de Barros MMM alverne, Quinderé PHD, Gondim LGF. Tecendo a rede assistencial em saúde mental com a ferramenta matricial. Physis. 2011;21(4):1579-99.

25. Dimenstein $M$, Severo $A K$, Brito $M$, Pimenta $A L$, Medeiros $V$, Bezerra E. $O$ apoio matricial em unidades de saúde da família: experimentando inovações em saúde. Saude Soc. 2009;18(1):63-74. https://doi.org/10.1590/S0104-12902009000100007.

26. Reeves S. Porque precisamos da educação interprofissional para um cuidado efetivo e seguro. Interface (Botucatu). 2016;20(56):185-96. https://doi.org/10.1590/180757622014.0092.

\section{Minicurrículo}

Judith Rafaelle Oliveira Pinho | https://orcid.org/0000-0001-8857-8138

http://lattes.cnpq.br/1541809941431244

Ivonice Siqueira de Sá | https://orcid.org/0000-0002-1800-8903

http://lattes.cnpq.br/1096757391975458

Christiana Leal Salgado | https://orcid.org/0000-0001-5547-7445

http://lattes.cnpq.br/1042489510713311

Paola Trindade Garcia | https://orcid.org/0000-0001-9105-4458

http://lattes.cnpq.br/8513290455922124

Ana Emília Figueiredo de Oliveira | https://orcid.org/0000-0003-4371-4815

http://lattes.cnpq.br/5767658067485112 